\title{
Investigating Temporal Implications of Information Transition in Submarine Command Teams
}

\author{
Kiome Pope ${ }^{1}$, Aaron Roberts ${ }^{1}$, and Neville Stanton ${ }^{1}$ \\ ${ }^{1}$ Human Factors Engineering, Transportation Research Group, Faculty of Engineering and \\ the Environment, University of Southampton, Boldrewood Campus, Burgess Road, \\ Southampton, SO16 7QF, UK. \\ \{K.A.Pope, apr1c13, N.Stanton\}@soton.ac.uk
}

\begin{abstract}
Previous work revealed bottlenecks in information transition between submarine command team members, which may be detrimental to overall performance. To date, the potential impact of such bottlenecks in terms of time to pass critical information has not been investigated. An understanding of the temporal impact is critical, given the time critical nature of tactical picture generation. Four teams of eight participants (32 participants total) were recruited and trained at one of the operator stations in a bespoke built submarine control room simulator. Participants completed high and low demand Return to Periscope Depth scenarios. The timings of technology-human and human-human interactions were calculated. Preliminary results indicated that the largest temporal lag was the transition of human-human information. Furthermore, loss of information occurred with every stage of information transition. As demand increased, the efficiency of information transition was negatively impacted by increased communications between operators. Initial results are discussed along with future analysis plans.
\end{abstract}

Keywords: Human Factors · Submarine · Command Team · Information Transition

\section{Introduction}

A submarine control room is an excellent example of a sociotechnical system where humans and technology interact in goal directed behaviors to achieve global team objectives [1]. In a submarine control room, information is distributed between operators and technology, which must be combined to form an accurate tactical picture [2, 3, 4]. Communications between members of the control room are crucial for effective integration of information from different sensors [5]. Data needs to be assimilated in a timely manner from various sources within the control room so that the Officer of the Watch (OOW) or Commanding Officer (CO) can make appropriate decisions for own ship safety [6, 7]. Delayed communications between command team members may affect the Situation Awareness (SA) of team members dependent on such information, leading to negative operational consequences [8]. A breakdown in communication between command team members regarding a new contact was a contributing factor in 
the collision of the USS Greeneville with the Ehime Maru, as the Officer of the Deck was not aware of the close range of the Ehime Maru [9].

The Command Team Experimental Test-bed (ComTET) project was a body of work that aimed to examine the current and future capabilities of a submarine control room. Previous work provided valuable insight into the interactions between members of a submarine command team [10, 2, 11, 12]. A shortened version of the Event Analysis for Systemic Teamwork (EAST) [11] method was used [10, 2, 11, 12], which modeled systems as a network of networks [2]. Social network analysis revealed a 'bottleneck' between the Operations Officer (OPSO) and the Sonar Controller (SOC) [12]. These operators communicated frequently in order to facilitate the building of an accurate tactical picture $[10,11,12]$. The SOC had to pass information (e.g. bearing and classification of surrounding vessels) from the sound room to the OPSO. The OPSO then passed this information to Target Motion Analysis (TMA) operators in the picture room so that they could build a tactical picture [11]. A 'bottleneck' between the OPSO and the SOC may cause a delay in transition of information from the sound room to the picture room, ultimately delaying the generation of a reliable tactical picture. The passage of information between the sound and picture room was highly reliant on the link between the OPSO and the SOC. It is likely that if this link were to be broken, a significant deterioration in performance would be observed [11] as the TMA operators would not have received the required information to form an accurate tactical picture. A noted limitation of the work by Roberts et al., [10] was the absence of temporal evaluation of social and task networks as scenarios progressed. Timely completion of tasks is important for safe and efficient operation; for example, taking too long to find a solution for a detected vessel may endanger own ship via counter-detection [4]. Thus, there is pressure to complete tasks quickly and accurately [14]. The current work aimed to build on the EAST analysis already conducted by the ComTET team [10,11, 12], to evaluate the impact of 'bottlenecks' on the speed of information transition and frequency of task completion. The following predictions were made.

Hypothesis 1: The slowest transition of information between operators will be between SOC and OPSO

Hypothesis 2: Loss of information will occur at each point of information transfer

Hypothesis 3: Information transfer will be slower in high demand conditions

\section{Method}

\subsection{Participants}

Ten teams of eight individuals have been recruited opportunistically from a variety of backgrounds, including undergraduate students and graduate recruits. A total of 71 males and 9 females participated with an age range of 18-55 (Mean $=26.83$, Standard Deviation $=8.69$ ). However, to date, four teams (32 participants total) have been processed for analysis, and thus their results are the focus of the current work. The study protocol received ethical approval from the University of Southampton Research Ethics Committee (Protocol No: 10099) and Ministry of Defense Research Ethics Committee (MoDREC) (Protocol No: 551/MODREC/14). 


\subsection{Equipment and Materials}

The ComTET team designed and built a submarine control room simulator based upon a currently operational Royal Navy submarine (see figure 1). Subject Matter Experts (SMEs) informed the design and build of the simulator and a full description of the process is provided by Roberts et al., [15]. The simulator had nine networked stations, each with a communications headset, a keyboard, two stacked monitors, and a mouse. A modified version of the Dangerous Waters (DW) simulation engine allowed the simultaneous completion of submarine command team tasks. The simulator had the capacity to record all verbal and non-verbal communications that occurred between operators. Recording software captured any transmissions over the communication network, and a collection of 10 web cameras, two high-resolution video cameras, and two ambient microphones captured all other communications. Screen recording software was used to record each individual screen in order to capture interactions with the technological system.

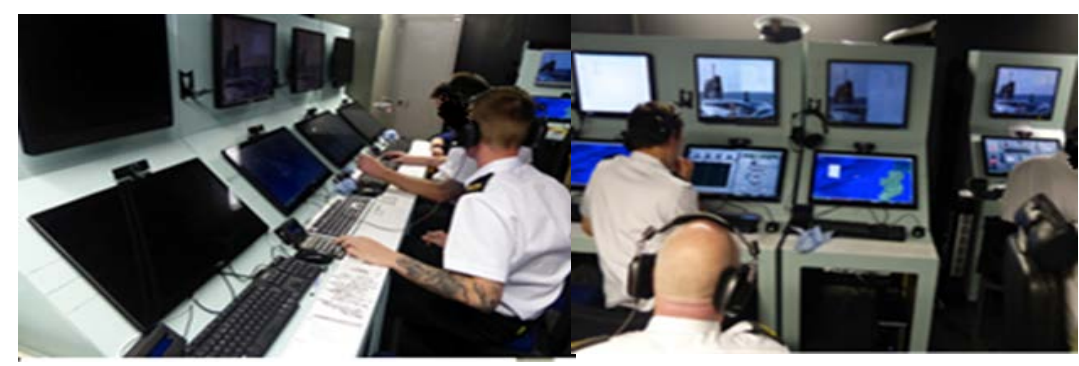

Fig. 1. The ComTET submarine control room simulator (a) left - control room (b) right - sound room

The operators chosen for inclusion were representative of a submarine command team; such selection was informed by SMEs. The operators included were an OOW, an OPSO, a SOC, two Sonar Operators (SOP), two TMA operators, a Periscope operator (PERI), and a Ship Control operator (SHC). A member of the ComTET team played the role of the OOW in order to guide teams tactically with relevant task fidelity.

Two Return to Periscope Depth (RTPD) scenarios were designed and programmed in DW (see Table 1 for a description). Scenario demand was manipulated by including a larger number of contacts with more erratic movements, and a second wave of contacts. Each scenario lasted approximately 45 minutes.

Table 1. Description of Scenarios

\begin{tabular}{lll}
\hline Demand & $\begin{array}{l}\text { No. Con- } \\
\text { tacts }\end{array}$ & Objective \\
\hline Low & 4 & $\begin{array}{l}\text { RTPD from deep in order to transmit intelligence home. } \\
\text { A large temporal window of opportunity is available. All } \\
\text { contacts held must be ranged so an optimum course for } \\
\text { RTPD can be found. Mission is complete once periscope } \\
\text { has been raised and all contacts marked. }\end{array}$ \\
& &
\end{tabular}




\begin{tabular}{ll}
\hline High 13 & $\begin{array}{l}\text { Due to severe submarine damage RTPD from deep as } \\
\text { quickly as possible. All contacts need to be ranged to find } \\
\text { an optimal route for RTPD. }\end{array}$ \\
\hline
\end{tabular}

To facilitate the recruitment of large numbers of participants, affording statistical robustness, a tutorial package was developed to train novice participants to be representative of a submarine command team. The training package consisted of six hourlong video tutorials, which covered essential concepts (e.g. bearing, course, range), and operator station specifics (e.g. Periscope tutorial). A communications game was also developed to train participants in military verbal protocol.

\subsection{Design}

This was a one factor repeated measures design. The independent variable was scenario demand. The dependent variables included time for human-human and technology-human information transition and frequency of tasks completed.

\subsection{Procedure}

Testing was conducted over two days; a training day and a testing day. On the training day, informed consent was obtained from participants and operator roles were randomly assigned. The morning consisted of general submarine command team training such as communication structure and essential concepts. In the afternoon, participants watched workstation specific tutorial videos and were allowed time to practice using the workstation before completing practice scenarios as part of a command team.

On the testing day, participants completed a final practice scenario monitored by the experimental team to ensure adequate performance of all tasks. Further training was provided if required. Participants then completed all scenarios. To prevent order effects the scenarios were counterbalanced across the four teams. Participants were told that the first scenario would begin, the recording devices were started, and the OOW gave a briefing. The scenario ended when the command team had completed the mission objective. After a debrief and a short break for refreshments, participants were asked to sit back at their workstation for the next scenario. At the end of the final scenario participants were given a full debrief and thanked for participating.

\subsection{Analysis of Data}

To complete the analysis raw data from individual screen recordings and web cameras were used to obtain time stamps for when both verbal and non-verbal tasks occurred. This was completed for all sonar and picture tasks and was conducted for all operators. The tasks included were identified from previous studies where SME input facilitated understanding of tasks undertaken in a submarine control room [10, 11, 12]. Individual scenario recordings were temporally aligned to ensure accurate task completion times between sources.

To date, not enough teams have been pre-processed to afford statistical analysis; however, this is intended in the future. Upon completion of recruitment and testing, it 
is intended that a repeated measures Analyses of Variances (ANOVA) will be conducted to examine differences between high and low demand scenarios. A sub-set of sonar tasks relating to contact designations are presented in the results section, for a description of each task see Table 2. Briefly, the SOPs must use passive broadband sonar to detect other vessels surrounding own ship [12, 16, 17]. This can be completed using the bow array, which is located at the front of own-ship, or using the hull (flank) array, which is located on the side [18]. Once detected a contact must be designated assigned a name such as "sonar zero one". This information must then be communicated to the SOC, who will then relay it to the OPSO [12]. The OPSO should then communicate this information to the TMA operators so that they can check that bearings cuts are being received for the contact. Knowledge of surrounding vessels is critical for team situation awareness [16] to avoid negative operational consequences [9]. Means and standard deviations for frequency of task completion and information transition are presented in the results along with the time taken.

Table 2. Description of Tasks Completed

\begin{tabular}{|c|c|c|}
\hline Task & Description & $\begin{array}{l}\text { Operators/Technologies in- } \\
\text { volved }\end{array}$ \\
\hline Designate bow contact & $\begin{array}{l}\text { Assign a name to a contact detected } \\
\text { on the bow array }\end{array}$ & SOP1/SOP2 and bow array \\
\hline Designate hull contact & $\begin{array}{l}\text { Assign a name to a contact detected } \\
\text { on the hull array }\end{array}$ & SOP1/SOP2 and hull array \\
\hline $\begin{array}{l}\text { Bow designation from } \\
\text { SOP to SOC }\end{array}$ & $\begin{array}{l}\text { SOP verbally passed designation in- } \\
\text { formation for contacts on the bow ar- } \\
\text { ray }\end{array}$ & SOP1/SO2 and SOC \\
\hline $\begin{array}{l}\text { Hull designation from } \\
\text { SOP to SOC }\end{array}$ & $\begin{array}{l}\text { SOP verbally passed designation in- } \\
\text { formation for contacts on the hull ar- } \\
\text { ray }\end{array}$ & SOP1/SOP2 and SOC \\
\hline $\begin{array}{l}\text { Designation from SOC to } \\
\text { OPSO }\end{array}$ & $\begin{array}{l}\text { SOC verbally passed designation in- } \\
\text { formation to OPSO that they re- } \\
\text { ceived from the SOPs }\end{array}$ & SOC and OPSO \\
\hline $\begin{array}{l}\text { SOC's own screen desig- } \\
\text { nation to OPSO }\end{array}$ & $\begin{array}{l}\text { SOC verbally passed designation in- } \\
\text { formation to OPSO that they re- } \\
\text { ceived from the SOPs }\end{array}$ & $\begin{array}{l}\text { SOC (using bow/hull ar- } \\
\text { ray) and OPSO }\end{array}$ \\
\hline $\begin{array}{l}\text { Designations from OPSO } \\
\text { to TMA }\end{array}$ & $\begin{array}{l}\text { OPSO verbally passed designation } \\
\text { information to the TMA operators }\end{array}$ & OPSO and TMA \\
\hline $\begin{array}{l}\text { TMA check cuts from } \\
\text { OPSO }\end{array}$ & $\begin{array}{l}\text { TMA checked cuts they verbally re- } \\
\text { ceived from OPSO }\end{array}$ & TMA and picture screen \\
\hline TMA check system cuts & $\begin{array}{l}\text { TMA checked cuts received via the } \\
\text { technological system }\end{array}$ & TMA and picture screen \\
\hline
\end{tabular}

\section{Results}

The ComTET team are in the process of completing the pre-processing and analysis of the temporal data for all teams, with the intention of completing statistical analysis. The current work presents results from four teams for RTPD high and low demand scenarios regarding designation of contacts to provide an early indication of the direction of the work. 
The number of contacts designated in the high demand was greater than in the low demand for both arrays. This was expected, given the greater number of contacts presented in the high demand scenario. However, the difference between the number of contacts designated on the hull array in high and low demand was small $(<1)$ compared to the bow array. The time taken to complete designations was slower in the high demand for both arrays. In both levels of demand, the designation of bow contacts was faster than the designation of hull contacts. This is despite fewer contacts designated on the hull array. It may be that teams prioritized the bow array over the hull array (see Table 3 for a summary of task frequency and duration).

The subsequent passage of designations to the SOC was completed with greater frequency in the high demand for both arrays. Again, this was to be expected due to the greater number of contacts presented in the high demand. However, in both levels of demand fewer designations were passed to the SOC than were designated, indicating task depletion between the SOPs and the SOC.

Fewer designations were passed from the SOC to the OPSO in the low demand than in the high demand. However, regardless of source, this was completed faster in the high demand, indicating that there may have been greater pressure to pass designations in a timely fashion. In both levels of demand, the SOC passed more designations from their own screen than those received from the SOPs. In the high demand, this information was transferred to the SOC faster by the technological system than by the SOPs, suggesting that the technological system was ahead of the human operators. Task depletion was also observed between the SOC and the OPSO, as the SOC passed fewer designations than they received from the SOPs, and fewer designations than they could observe from their own screens.

The OPSO passed more designations to the TMA operators in the low demand, but completed the transfer of information faster in the low demand than in the high demand. Fewer designations were passed to the TMA operators than the OPSO received, indicating further task depletion. The task of TMA operators checking cuts from OPSO was only completed in the low demand scenario, and cuts received via the technological system were checked with higher frequency for both demand levels. The frequency of cuts checked from the system was greater in the high demand, in line with the greater number of contacts presented. This was completed slower in the high demand.

Table 3. Frequency and Average Time of Task Completion in RTPD Scenarios at high and low workloads

\begin{tabular}{|c|c|c|c|c|}
\hline \multirow[b]{2}{*}{ Task } & \multicolumn{2}{|c|}{ Frequency of Completion } & \multicolumn{2}{|c|}{ Average Time for Completion } \\
\hline & Low & High & Low & High \\
\hline$\overline{\text { Designate bow contact }}$ & $4.00 \pm .00$ & $11.00 \pm 1.63$ & $02: 41 \pm 01: 34$ & $05: 26 \pm 05: 24$ \\
\hline Designate hull contact & $3.75 \pm .50$ & $4.50 \pm 1.91$ & $05: 46 \pm 05: 21$ & 07:21 \pm 06:05 \\
\hline $\begin{array}{l}\text { Bow designation from } \\
\text { SOP to SOC }\end{array}$ & $.94 \pm 1.30$ & $5.50 \pm 4.12$ & 00:09 $\pm 00: 13$ & 01:07 \pm 01:00 \\
\hline $\begin{array}{l}\text { Hull designation from } \\
\text { SOP to SOC }\end{array}$ & $1.75 \pm 1.71$ & $3.00 \pm 2.58$ & $02: 18 \pm 01: 42$ & $00: 31 \pm 00: 19$ \\
\hline $\begin{array}{l}\text { Designation from SOC } \\
\text { to OPSO }\end{array}$ & $1.50 \pm 1.73$ & $1.75 \pm 1.71$ & 07:24 $\pm 01: 14$ & 02:51 \pm 03:06 \\
\hline $\begin{array}{l}\text { SOC's own screen } \\
\text { designation to OPSO }\end{array}$ & $2.50 \pm 1.29$ & $2.25 \pm 2.22$ & $03: 43 \pm 06: 14$ & $02: 24 \pm 02: 04$ \\
\hline
\end{tabular}




\begin{tabular}{|c|c|c|c|c|}
\hline $\begin{array}{ll}\text { Designations } & \text { from } \\
\text { OPSO to TMA } & \end{array}$ & $1.00 \pm 2.00$ & $.50 \pm .58$ & $00: 30 \pm 00: 11$ & $13: 58 \pm 13: 48$ \\
\hline $\begin{array}{l}\text { TMA check cuts from } \\
\text { OPSO }\end{array}$ & $1.00 \pm 2.00$ & $.00 \pm .00$ & $00: 48$ & - \\
\hline $\begin{array}{l}\text { TMA check system } \\
\text { cuts }\end{array}$ & $9.50 \pm 2.52$ & $20.50 \pm 5.00$ & $04: 25 \pm 05: 42$ & $05: 54 \pm 05: 32$ \\
\hline
\end{tabular}

Note. Means and Standard Deviations presented for frequency of completion and time for completion. Some key results highlighted.

A case study of a RTPD high demand scenario is presented in figure 2. Square boxes represent technological interfaces and circles represent operators in the system. Contacts were designated faster and with greater frequency on the bow array than the hull array, potentially indicating a prioritization of the bow array.

Subsequent information transfer was faster for bow designations; however, more hull designations were verbally passed to the SOC. Thus, greater task depletion was seen between the SOPs and the SOC for the bow array. Task depletion also occurred between the SOC and the OPSO, as only two of the six designations received by the SOC were transferred to the OPSO. Similarly, of the 17 designations that would have been visible on the SOCs screen, only three were passed to the OPSO from SOC's own screen. The transfer of information from the SOC's own screen was faster than the transfer of designations received from the SOPs. The OPSO did not pass any designation information to the TMA operators; rather cuts were checked via the technological system. This indicates task depletion between the OPSO and TMA operators.

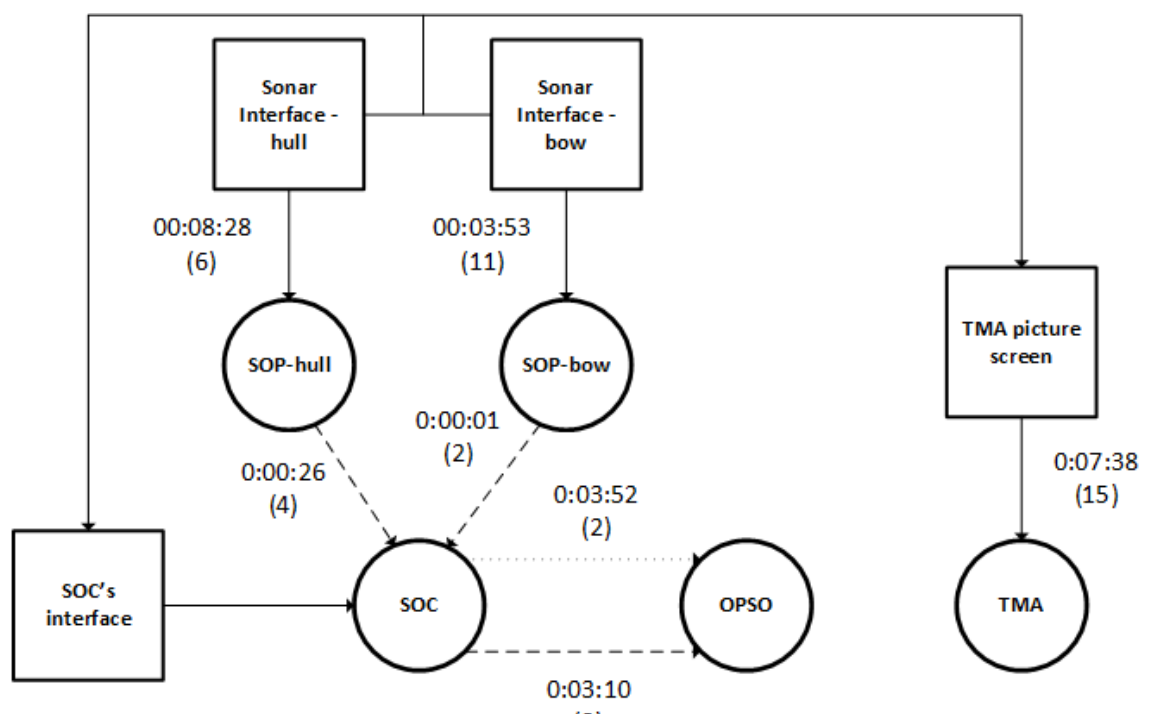

(3)

Fig 2. Case study of one team RTPD high - solid lines represent interactions with the technological system, dashed lines represent verbal communication of information received from the technological system, and dotted lines represent information received from other operators 


\section{Discussion}

The current work aimed to evaluate the impact of 'bottlenecks' on the speed of information transition and frequency of task completion through the control room. The results provided tentative support for the hypotheses proposed. In the low demand scenario, the slowest transition of information was between the OPSO and the SOC. In the high demand, the first hypothesis was not supported, as the slowest transition was between the OPSO and TMA operators. However, in the case study, this information transition did not occur, and thus the slowest transition of information was between the OPSO and the SOC. Overall it is evident that there is a delay in information transition between the OPSO and the SOC. The second hypothesis was supported by the results. At each point in the network, less information was passed than was received, regardless of source of information. This may represent the quality checking and refinement of information being passed through the command team. However, it may also reflect the loss of information due to excessive workload, which may have a negative effect on safety. The third hypothesis was not supported, as generally the transition of information was faster in the high demand. This may be indicative of increased pressure to pass information in a timely manner [14].

Despite a greater number of contacts presented in the high demand scenario, a similar number of contacts were designated on the hull array in both levels of demand. Previous work by the ComTET team found that scenario demand significantly affected subjective measures of mental and temporal load [19]. It may be that SOPs' workload was so great in the high demand that they were unable to work on the hull array, and instead prioritized the bow array. Consequently, contacts on the hull array may have been missed, and if not picked up on the bow array, this can endanger own ship. Similar usage of the different arrays was observed in the case study, with fewer contacts designated on the hull array. Further evidence of the high demand placed on the SOPs resulted in the usage of the SOC's own screen to pass information to the OPSO. In the case study, the passage of information from the SOC's interface was faster than those received from the SOPs. This may be beneficial for information regarding priority contacts, as the information can be transferred to the OPSO faster. However, this may be detrimental for shared situation awareness as knowledge gained by the SOPs from the sonar displays is not communicated, and instead the information transition is reliant on the SOCs' interpretation. There is pressure to complete tasks quickly and accurately in a submarine command room [14], which may have led to the SOC utilizing their own screen rather than waiting for the SOPs to pass the required information. Slow information transition, and task depletion occurred between the SOC and the OPSO, a bottleneck previously identified by the ComTET team from social network analysis [10, $11,12]$. In the current case study, only a third of the information verbally received by the SOC was transferred to the OPSO and the information was transferred far slower than received. This means that the transition of vital information may be delayed or information may not be communicated at all. This can potentially compromise own ship safety, as there is a lack of shared awareness regarding vessels surrounding own ship.

The slowest transfer of information was between the OPSO and the TMA operators in the high demand scenario. However, designation information could be transferred via the technological system to the TMA operators, as well as via operators. Information 
was transferred faster by the technological system than via operators in the control room. This could lead to the TMA operators being ahead of the command structure (the OOW, OPSO, \& SOC) with regard to awareness of surrounding vessels. This could lead to unnecessary confusion, as operators may not have received the necessary guidance on contact prioritization and workload delegation. In the case study, no information was transferred by the OPSO to the TMA operators, meaning that information was received faster via the technological system (as opposed to not at all). This may have negative consequences for situation awareness, as the OPSO would not have been aware if cuts were not being received. Furthermore, the OPSO may not have been aware of what each operator was working on; this may have resulted in duplication of work by the TMA operators or focusing on low priority contacts, hindering the formation of an accurate tactical picture.

The current research builds upon previous work conducted by the ComTET team, investigating current ways of working in a submarine control room [10,11, 12]. In the current work, the social and task networks are combined to provide a temporal understanding of task completion and information transition. Furthermore, this work examines the interactions of operators with the technological system, an aspect that was not examined in previous work [10].

\subsection{Conclusions and future work}

Submarine crews of the future will be required to process large volumes of data with similar or potentially reduced crew sizes [15]. Larger volumes of data with the same crew sizes will require different team structures and allocation of tasks, thus an understanding of current ways of working is essential [15]. If temporal delay issues are evident in the current configuration, then an examination of new configurations is needed, as the addition of more sensors, which generate more data, has the potential to compound these problems further. The current work highlights the impact of the 'bottleneck' between the OPSO and the SOC on the speed of information transition across the control room. It is recommended that reliance on communication between the OPSO and the SOC is reduced, to facilitate better flow of information through the command team. Co-locating operators dependent on each other for task relevant information may achieve this. For example, the TMA operators routinely require information from the SOPs in order to complete tasks. Thus, allowing these operators to communicate directly may allow for faster transition of information across the command team. However, such recommendations need to be evidence based. It is for such reasons the current work will be extended to include ten teams, affording robust statistical comparisons.

\section{References}

1. Walker, G.H., Stanton, N.A., Salmon, P.M., Jenkins, D.P.: A review of sociotechnical systems theory: a classic concept for new command and control paradigms. Theoretical Issues in Ergonomics Science, 9, 6, 479-499 (2008).

2. Stanton, N.A.: Representing distributed cognition in complex systems: how a submarine returns to periscope depth. Ergonomics, 57, 3, 403-418 (2014). 
3. Jones, E., Steed, R., Diedrich, F., Armbruster, R., Jackson, C.: Performance-based metrics for evaluating submarine command team decision making. Foundations of Augmented Cognition. Directing the Future of Adaptive Systems, 308-317 (2011).

4. Kirschenbaum, S.S., Trafton, J.G., Schunn, C.D., Trickett, S.B.: Visualizing uncertainty: the impact on performance. Human Factors, 56, 3, 509-520 (2014).

5. Huf, S., French, H.T.: Situation awareness in a networked virtual submarine. In Proceedings of the human factors and ergonomics society annual meeting, 48, 3, 663-667 (2004).

6. Dominguez, C., Long, W.G., Miller, T.E., Wiggins, S.L.: Design directions for support of a submarine commanding officer decision making. Paper presented at the Proceedings of 2006 Undersea HSI Symposium: Research, Acquisition and the Warrior (2006).

7. Duryea, D.M., Lindstrom, C.E., Sayegh, R.: Submarine imaging systems: developing improved capabilities and technologies. Paper presented at the Sensors, and Command, Control, Communications, and Intelligence (C3I) Technologies for Homeland Security and Homeland Defense VII (2008).

8. Salas, E., Burke, C.S., Samman, S.N.: Understanding command and control teams operating in complex environments. Information Knowledge Systems Management, 2, 4, 311-323 (2001).

9. Roberts, K.H., Tadmor, C.T.: Lessons learned from non-medical industries: the tragedy of the USS Greeneville. Qual Saf Health Care, 11, 4, 355-357 (2002).

10. Roberts, A.P.J, Stanton, N.A., Fay, D.: Land ahoy! Understanding submarine command and control during the completion of inshore operations. Human Factors, 59, 8, 1263-1288 (2017).

11. Stanton, N.A., Roberts, A.P.J: Examining social, information, and task networks in submarine command and control. IEEE Transactions on Human Machine Systems (2017).

12. Stanton, N.A., Roberts, A.P.J., Fay D.T.: Up periscope: understanding submarine command and control teamwork during a simulated return to periscope depth. Cognition, Technology and Work, 19, 2-3, 399-417 (2017).

13. Stanton, N.A., Baber, C., Harris, D.: Modelling command and control: Event analysis of systemic teamwork. Aldershot, UK: Ashgate (2012).

14. Hautamaki, B.S., Bagnall, T., Small, R.: Human interface evaluation methods for submarine combat systems. Retrieved from https://pdfs.semanticscholar.org/1291/032fa76168f05599b31dcf3671e2fe7a9486.pdf (2006).

15.Roberts, A., Stanton, N.A., Fay. D.: The command team experimental test-bed stage 1: design and build of a submarine command room simulator. Procedia Manufacturing, 3, 2800-2807 (2015).

16. Zarnich, R. E.: A fresh look at broadband passive sonar processing. Adaptive sensor array processing workship, Office of Naval Research, 99-104 (1999).

17. Shar, P., Li, X.R.: Passive sonar fusion for submarine $C^{2}$ systems. IEEE Aerospace and Electronic Systems Magazine, 15, 3, 29-34 (2000).

18. Baggeroer, A.B.: Sonar arrays and array processing. AIP Conference Proceedings, 760, 3, (2005).

19. Roberts, A., Stanton, N.A., Fay, D.: The command team experimental test-bed phase two: assessing cognitive load and situation awareness in a submarine control room. Advances in Human Aspects of Transportation, Springer, 427-437 (2017). 